\title{
A comparative study of nickel-zinc ferrites by sol-gel route and solid-state reaction
}

\begin{abstract}
The properties of ferrites are affected by the microstructural problems which have become the most serious obstacles in obtaining high quality reproducible ferrites. In this study, the nickel-zinc ferrites were prepared via two different methods: the conventional classical ceramic method known as the solid-state reaction, and the newer sol-gel method. The electromagnetic and microstructural properties of both different samples were also discussed. A higher electrical resistivity and controlled initial permeability with a smaller loss were found in the sol-gel samples. Consequently, the homogenous microstructure and the advantages in terms of powder and sample preparations have been discovered by means of sol-gel technique.
\end{abstract}

Keyword: Sol-gel growth; Magnetic materials; Sintering; Microstructure 